\title{
Comparison of somnolence associated with asenapine, olanzapine, risperidone, and haloperidol relative to placebo in patients with schizophrenia or bipolar disorder
}

\author{
This article was published in the following Dove Press journal: \\ Neuropsychiatric Disease and Treatment \\ 21 August 2013 \\ Number of times this article has been viewed
}

\section{Keming Gao' \\ Mary Mackle² \\ Pilar Cazorla ${ }^{2}$ \\ Jun Zhao ${ }^{2}$}

Armin Szegedi ${ }^{2}$

'Department of Psychiatry, Mood and Anxiety Clinic in the Mood Disorders Program, Case Western Reserve University, School of Medicine, Cleveland, OH, USA; ${ }^{2}$ Merck, Rahway, NJ, USA
Correspondence: Keming Gao Department of Psychiatry, Mood and Anxiety Clinic in the Mood Disorders Program, Case Western Reserve University, School of Medicine, Cleveland, $\mathrm{OH}$, USA

Tel +I 2168442656

Fax + I 2168442875

Email keming.gao@uhhospitals.org
Background: Patients with schizophrenia or bipolar disorder (BPD) may be differentially sensitive to antipsychotics. This study assessed the median time to onset, duration, and rate of somnolence associated with asenapine and other antipsychotics in both indications.

Methods: Ten clinical trials $(n=4786)$ were analyzed as five cohorts pooled according to indication and study design.

Results: In the short-term schizophrenia cohort, the incidence of somnolence was $13.1 \%, 19.1 \%$, $8.5 \% 5.2 \%$, and $6.9 \%$ with asenapine, olanzapine, risperidone, haloperidol, and placebo, respectively. Median time to onset of somnolence was 2 days for asenapine and olanzapine, and 6, 3, and 7 days for risperidone, haloperidol, and placebo, respectively. Median duration was 15 days for asenapine and olanzapine, and 3, 22.5, and 4.5 days for risperidone, haloperidol, and placebo, respectively. In the long-term schizophrenia cohort, the incidence, time to onset, and duration of somnolence with asenapine and olanzapine were $18.4 \%$ versus $19.6 \%, 9.0$ days versus 12 days, and 22 days versus 21 days, respectively. In schizophrenia with persistent negative symptoms, the incidence, median time to onset, and duration of somnolence with asenapine and olanzapine were $18.5 \%$ versus $21.1 \%, 9.0$ days versus 7.5 days, and 25.0 days versus 41.5 days, respectively. In the monotherapy for BPD cohort, the incidence of somnolence with asenapine, olanzapine, and placebo was $23.8 \%, 26.4 \%$, and $6.4 \%$, respectively. Median time to onset and duration of somnolence with asenapine, olanzapine, and placebo were 1,2, and 2 days, respectively, and 7 , 8.5 , and 5 days. In the adjunctive therapy for BPD cohort, the incidence, median time to onset, and duration of somnolence with asenapine and placebo were $24.0 \%$ versus $10.2 \%, 1.5$ days versus 2 days, and 12.5 days versus 7 days, respectively.

Conclusion: In the short-term schizophrenia cohort, time to onset and duration of somnolence with asenapine was similar to that with olanzapine and haloperidol. Only asenapine and olanzapine had significantly higher rates of somnolence relative to placebo. The time to onset, duration, and incidence of somnolence with asenapine and olanzapine was similar in patients with long-term schizophrenia and those with BPD. Patients with BPD were more sensitive than those with schizophrenia to asenapine and olanzapine.

Keywords: asenapine, somnolence, sedation, schizophrenia, bipolar disorder

\section{Introduction}

Schizophrenia and bipolar disorder (BPD) are two of the most common and debilitating psychiatric disorders, causing severe impairment, and are associated with an increased risk of suicide. ${ }^{1}$ Although each disorder presents with its own distinct symptom profile, antipsychotics are commonly used in both conditions. Depending on the 
pharmacological profile of a drug, the use of antipsychotics in patients with schizophrenia or BPD can be associated with an increased incidence of somnolence, including sedation and hypersomnia. ${ }^{2}$ Antipsychotic-induced sedation can be associated with poor cognition and an increase in reported accidents, and prolonged or severe sedation could potentially impair quality of life and result in noncompliance with medication. However, sedation may be helpful under certain circumstances in the management of acute schizophrenia or mania because of its ability to regulate sleep disturbances and manage agitation. In this regard, patients with BPD have been reported to be more sensitive in general than those with schizophrenia to the sedative effects of antipsychotics. ${ }^{2}$

Asenapine is a tetracyclic antipsychotic with a unique pharmacologic profile, ${ }^{3}$ and is approved in the United States for the treatment of schizophrenia in adults and for the acute treatment of manic or mixed episodes associated with bipolar I disorder, either as monotherapy or as therapy adjunctive to lithium or valproate. In the European Union, asenapine is approved for the treatment of moderate to severe manic episodes associated with bipolar I disorder. ${ }^{4}$ In both short-term and long-term clinical studies, sublingual asenapine was effective and generally well tolerated in patients with schizophrenia ${ }^{5-8}$ and in those with manic or mixed symptoms associated with bipolar I disorder. ${ }^{9-13}$

Asenapine is formulated as a fast dissolving and rapidly absorbed sublingual tablet, and is able to be initiated at therapeutic doses without titration. Like some antipsychotics, ${ }^{2}$ somnolence and/or sedation are the most commonly reported adverse events associated with asenapine in patients with schizophrenia or BPD. ${ }^{14}$ However, it remains unclear if asenapine has a similar risk of treatment-emergent somnolence compared with other antipsychotics, and if patients with schizophrenia or BPD have a similar risk, onset, and duration of treatment-emergent somnolence on asenapine. Such information would aid clinicians in the appropriate use of asenapine. Therefore, we conducted a post hoc analysis of 10 clinical trials of asenapine in the treatment of schizophrenia and BPD to compare the incidence, median time to onset, and average duration of somnolence/ sedation, to determine whether there was a difference in the risk of treatment-emergent somnolence with asenapine, olanzapine, risperidone, and haloperidol relative to placebo, and to explore if there was any difference in sensitivity to the sedative effects of asenapine in these therapeutic settings.

\section{Materials and methods}

This was a post hoc analysis of 10 clinical trials involving a total of 4786 patients. The data were analyzed and pooled into five cohorts according to treatment indication (cohorts 1, 2, and 3 for schizophrenia and cohorts 3 and 4 for bipolar mania). These five cohorts were also constructed based on study design, such as acute (cohort 1) versus long-term (cohorts 2 and 3), placebo-controlled (cohort 1) versus head-to-head active drug comparison (cohorts 2 and 3 ), fixeddose (cohort 1) versus flexible dose (cohorts 2 and 3), and monotherapy (cohort 4) versus adjunctive therapy (cohort 5). A summary description of each of the five cohorts, including study designs, patient population, permitted concomitant medications, and major eligibility criteria is presented in Table 1. In each study, patients assigned to asenapine received the study drug at a dose of 5-10 mg twice daily. The primary results of these studies have been reported elsewhere. ${ }^{5,7-9,12,13,15}$

For the purposes of this analysis, somnolence was defined as any treatment-emergent adverse event corresponding to the Medical Dictionary for Regulatory Activities (MedDRA) preferred terms of somnolence, sedation, or hypersomnia. The severity (maximum intensity of the event) was determined by the primary investigators of each study. If more than one event occurred, the maximum intensity was determined as the greatest of those events. The time to onset of somnolence was calculated as the number of days from the first dose of study medication to the first day of the event. Duration of somnolence was calculated as the number of days between the day of onset and the last day of the event. If an event was unresolved at the end of the study, the last day was imputed as seven days after the last dose for an adverse event or 30 days after the last dose for a serious adverse event. If there were no events by the end of the trial or at early discontinuation, the time to the event was considered right censored at the last day of the study medication.

\section{Schizophrenia}

\section{Cohort I: short-term schizophrenia}

The short-term schizophrenia cohort consisted of 1318 patients with acute exacerbation of their positive symptoms enrolled in four 6-week monotherapy trials, including 572 patients treated with asenapine (5-10 mg twice daily), 378 treated with placebo, 59 with risperidone (3 $\mathrm{mg}$ twice daily), 194 with olanzapine (5-20 mg once daily), and 115 with haloperidol (4 mg twice daily). 5,7,16

\section{Cohort 2: long-term schizophrenia and schizoaffective disorder}

The long-term (52-week) schizophrenia and schizoaffective cohort consisted of 1219 patients with acute exacerbation of 


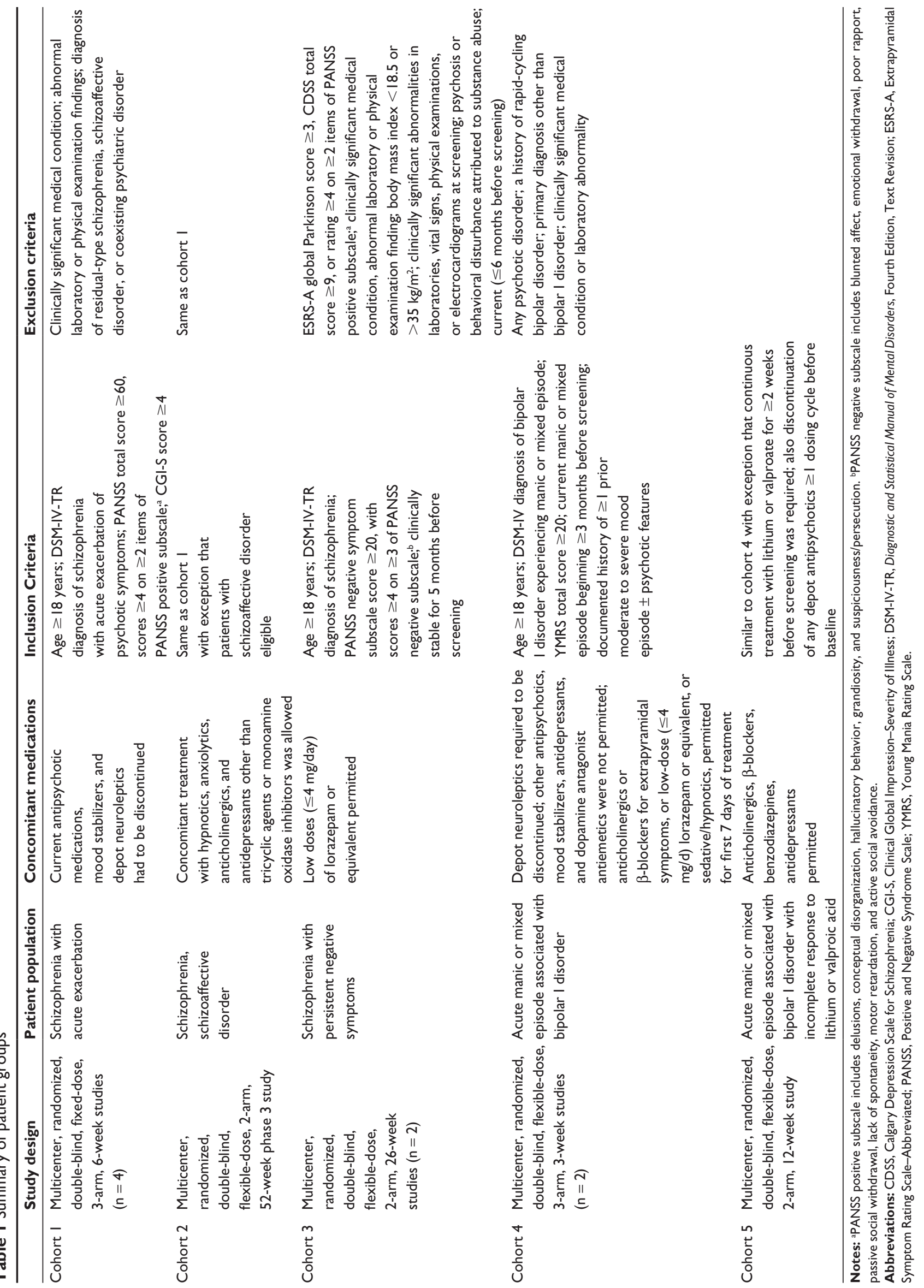


their positive symptoms and enrolled in a single monotherapy safety trial, including 908 asenapine-treated (5-10 mg twice daily) and 311 olanzapine-treated (5-20 mg once daily) patients. ${ }^{8}$

\section{Cohort 3: monotherapy for schizophrenia} with persistent negative symptoms

The persistent negative symptoms (PNS) cohort consisted of 949 patients suffering from PNS enrolled in two 26-week monotherapy trials, including 485 asenapine-treated (5-10 mg twice daily) and 464 olanzapine-treated (5-20 mg once daily) patients. ${ }^{15}$

\section{Bipolar mania}

Cohort 4: short-term monotherapy

for bipolar I disorder

The short-term bipolar I disorder cohort consisted of 976 patients enrolled in two 3-week monotherapy trials, including 379 patients treated with asenapine (5-10 mg twice daily), 203 with placebo, and 394 with olanzapine (5-20 mg once daily). ${ }^{9,12}$

\section{Cohort 5: short-term adjunctive therapy for bipolar I disorder}

The adjunctive bipolar I disorder cohort consisted of 324 patients enrolled in a single 12-week trial, including 158 asenapine-treated (5-10 mg twice daily) patients and 166 placebo-treated patients who were administered asenapine as an adjunct to lithium or valproate. ${ }^{13}$

\section{Key outcomes}

Key outcome measures were incidence, time to onset, and duration of somnolence. Somnolence as an adverse event was defined using MedDRA preferred terms and included somnolence, sedation, and hypersomnia. An additional exploratory measure was the percentage of patients who took any sedatives/hypnotics or benzodiazepines during the studies and in whom use of these medications was associated with onset of somnolence within 24 hours.

\section{Subgroup analyses}

A subgroup analysis of patients in the short-term schizophrenia and short-term bipolar 1 disorder monotherapy cohorts (cohorts 1 and 4, respectively) was evaluated according to baseline Positive and Negative Syndrome Scale (PANSS) anxiety score G2 $<4$ or $\geq 4$, PANSS depression score G6 $<4$ or $\geq 4$, and PANSS Marder Factor (MF) anxiety/ depression score $<16$ or $\geq 16$. The BPD subgroups were evaluated using a Montgomery-Åsberg Depression Rating Scale (MADRS) total score $<20$ or $\geq 20$ and a Clinical Global Impressions of Bipolar Disorder severity of depression score $<4$ or $\geq 4$.

\section{Statistical analysis}

All post hoc analyses were conducted in patients who received at least one dose of active agent or placebo for each trial. Data were pooled across doses in each cohort for asenapine, active controls (risperidone, olanzapine, and haloperidol), and placebo. The number needed to treat to harm (NNH) for an event of somnolence for each active group relative to the placebo group was calculated in cohorts 1,4 , and 5, and relative to the olanzapine group in cohorts 2 and 3 when differences in the incidence rates between the treatment groups were statistically significant. The 95\% confidence intervals (CIs) were based on differences in treatment rate using the Miettinen and Nurminen method. ${ }^{17}$ Median time to onset and duration of somnolence were calculated based on patients who experienced treatment-emergent somnolence/ sedation events. $P$ values to test differences in distribution of time to onset of somnolence were calculated using the log-rank test.

\section{Results}

\section{Schizophrenia}

Cohort I: short-term schizophrenia risk for somnolence In the short-term schizophrenia cohort, the incidence of somnolence (Table 2) with all doses of asenapine was $13.1 \%$, 6.9\% with placebo, $8.5 \%$ with risperidone, $5.2 \%$ with haloperidol, and $19.1 \%$ with olanzapine. The NNH $(95 \% \mathrm{CI})$ for asenapine and olanzapine relative to placebo was $16(10,43)$ and $8(5,15)$, respectively. The difference between risperidone or haloperidol and placebo was not significant. No patients discontinued because of somnolence/sedation in the placebo, risperidone, or haloperidol groups, but one patient $(0.2 \%)$ receiving asenapine and two patients $(1.0 \%)$ receiving olanzapine discontinued due to somnolence. One event of severe somnolence was reported in the asenapine group. Moderate events of somnolence with placebo, asenapine, risperidone, olanzapine, and haloperidol were experienced by five $(1.3 \%), 13(2.3 \%)$, two $(3.4 \%), 13(6.7 \%)$, and one $(0.87 \%)$ patients, respectively (Figure 1). All other events of somnolence with each treatment were mild.

As shown in Table 2, in patients with less anxiety at baseline (PANSS G2 <4), only olanzapine had a significantly increased risk for somnolence, with a $\mathrm{NNH}$ 
Table 2 Somnolence in short-term schizophrenia cohort

\begin{tabular}{|c|c|c|c|c|}
\hline & Treatment & $\begin{array}{l}\text { Incidence } \\
\mathrm{n} / \mathrm{N}(\%)\end{array}$ & $\begin{array}{l}\text { Difference between active treatment } \\
\text { and placebo } \%(95 \% \mathrm{Cl})\end{array}$ & $\begin{array}{l}\text { NNH } \\
(95 \% \mathrm{Cl})\end{array}$ \\
\hline \multirow[t]{5}{*}{ Full sample } & Asenapine 5-10 mg twice daily & $75 / 572(13.1)$ & $6.23(2.34,9.98)$ & $16(10,43)$ \\
\hline & Risperidone $3 \mathrm{mg}$ twice daily & $5 / 59(8.5)$ & $1.60(-4.16,11.7)$ & ns \\
\hline & Olanzapine $5-20 \mathrm{mg}$ once daily & $37 / 194(19.1)$ & $12.19(6.49,18.73)$ & $8(5,15)$ \\
\hline & Haloperidol 4 mg twice daily & $6 / 115(5.2)$ & $-1.66(-5.88,4.40)$ & ns \\
\hline & Placebo & $26 / 378(6.9)$ & - & - \\
\hline \multirow[t]{5}{*}{$\mathrm{G} 2<4$} & Asenapine $5-10 \mathrm{mg}$ twice daily & $33 / 331(10.0)$ & $4.01(-0.80,8.52)$ & ns \\
\hline & Risperidone 3 mg twice daily & $4 / 27(14.8)$ & $8.85(-0.92,26.70)$ & ns \\
\hline & Olanzapine $5-20 \mathrm{mg}$ once daily & $18 / 100(18.0)$ & I2.04 (4.68, 2I.|4) & $8(5,2 I)$ \\
\hline & Haloperidol 4 mg twice daily & $0 / 78(0)$ & $-5.96(-9.94,-1.10)$ & ns \\
\hline & Placebo & $13 / 2 \mid 8(6.0)$ & - & - \\
\hline \multirow[t]{5}{*}{$\mathrm{G} 2 \geq 4$} & Asenapine 5-10 mg twice daily & $42 / 24 \mid(17.4)$ & $9.30(2.60,15.68)$ & II $(6,39)$ \\
\hline & Risperidone $3 \mathrm{mg}$ twice daily & $1 / 32(3.1)$ & $-5.00(-11.23,7.90)$ & ns \\
\hline & Olanzapine $5-20 \mathrm{mg}$ once daily & $19 / 94(20.2)$ & $12.09(3.50,22.03)$ & $8(5,29)$ \\
\hline & Haloperidol 4 mg twice daily & $6 / 37(16.2)$ & $8.09(-2.01,23.50)$ & ns \\
\hline & Placebo & $13 / 160(8.1)$ & - & - \\
\hline \multirow[t]{5}{*}{$M F<16$} & Asenapine 5-10 mg twice daily & $64 / 518(12.4)$ & $5.57(1.52,9.45)$ & $18(11,66)$ \\
\hline & Risperidone $3 \mathrm{mg}$ twice daily & $5 / 5 \mid(9.8)$ & $3.02(-3.45,14.40)$ & ns \\
\hline & Olanzapine $5-20 \mathrm{mg}$ once daily & $35 / 178(19.7)$ & $12.88(6.86,19.77)$ & $8(5,15)$ \\
\hline & Haloperidol 4 mg twice daily & $4 / 108(3.7)$ & $-3.08(-7.12,2.73)$ & ns \\
\hline & Placebo & $23 / 339(6.8)$ & - & - \\
\hline \multirow[t]{5}{*}{$M F \geq 16$} & Asenapine 5-10 mg twice daily & I I/54 (20.4) & $12.68(-2.46,26.76)$ & ns \\
\hline & Risperidone 3 mg twice daily & $0 / 8(0)$ & $-7.69(-20.50,25.70)$ & ns \\
\hline & Olanzapine $5-20 \mathrm{mg}$ once daily & $2 / 16(12.5)$ & $4.81(-11.15,29.00)$ & ns \\
\hline & Haloperidol 4 mg twice daily & $2 / 7(28.6)$ & $20.88(-3.06,57.60)$ & ns \\
\hline & Placebo & $3 / 39(7.7)$ & - & - \\
\hline \multirow[t]{5}{*}{$\mathrm{G} 6<4$} & Asenapine $5-10 \mathrm{mg}$ twice daily & $51 / 428(11.9)$ & $5.99(1.61,10.15)$ & $17(10,62)$ \\
\hline & Risperidone $3 \mathrm{mg}$ twice daily & $4 / 40(10.0)$ & $4.07(-2.97, \mid 17.30)$ & ns \\
\hline & Olanzapine $5-20 \mathrm{mg}$ once daily & $32 / 145(22.1)$ & $16.14(9.32,23.99)$ & $6(4,11)$ \\
\hline & Haloperidol 4 mg twice daily & $4 / 93(4.3)$ & $-1.62(-6.11,4.98)$ & ns \\
\hline & Placebo & $16 / 270(5.93)$ & - & - \\
\hline \multirow[t]{5}{*}{$\mathrm{G} 6 \geq 4$} & Asenapine 5-10 mg twice daily & $24 / 144(16.7)$ & $7.4 \mid(-1.22,15.68)$ & ns \\
\hline & Risperidone $3 \mathrm{mg}$ twice daily & $1 / 19(5.3)$ & $-4.00(-12.7|| 15.00)$, & ns \\
\hline & Olanzapine $5-20 \mathrm{mg}$ once daily & $5 / 49(10.2)$ & $0.94(-8.27,13.30)$ & ns \\
\hline & Haloperidol 4 mg twice daily & $2 / 22(9.1)$ & $-0.17(-10.11,19.00)$ & ns \\
\hline & Placebo & $10 / 108(9.3)$ & - & - \\
\hline
\end{tabular}

Abbreviations: $\mathrm{Cl}$, confidence interval; $\mathrm{N}$, total number of patients in treatment arm; $\mathrm{n}$, number of patients with treatment-emergent somnolence; \%, percentage of patients in treatment arm with treatment-emergent somnolence; G2, PANSS G2 (anxiety); MF, PANSS MF anxiety/depression; G6, PANSS G6 (depression); NNH, number needed to treat to result in an adverse event of somnolence; (harm) ns, not statistically significant; PANSS, Positive and Negative Syndrome Scale.

of $8[95 \% \mathrm{CI}(5,21)]$. In contrast, in patients with elevated baseline anxiety (PANSS G2 $\geq 4$ ), both asenapine and olanzapine were associated with a significantly increased risk of somnolence, with an NNH of 11 [95\% CI $(6,39)]$ and $8[95 \%$ CI $(5,29)]$, respectively. In patients with less severe depression at baseline (PANSS MF anxiety/ depression $<16$ or PANSS G6 $<4$ ), both asenapine and olanzapine had a significantly increased risk of somnolence, but in patients with more severe depression (PANSS MF anxiety/ depression $\geq 16$ or PANSS G6 $\geq 4$ ), none of the active treatments increased the risk of somnolence.

The median time to onset of somnolence (Figure 2A) was significantly shorter with asenapine (2.0 days; $P<0.01)$ and olanzapine (2.0 days; $P<0.0001)$ compared with placebo (7.0 days). The median times to onset with risperidone (6.0 days) and haloperidol (3.0 days) were not significantly different from placebo. The median duration of somnolence (Figure 3) with asenapine was numerically longer (15 days) than with placebo (4.5 days) or risperidone (3 days), similar to olanzapine (15 days), and numerically less than haloperidol (22.5 days). Duration of somnolence was longer in patients in the olanzapine group with elevated baseline anxiety (PANSS $\mathrm{G} 2 \geq 4$, Figure 4A). In the asenapine and olanzapine groups, the median duration of somnolence was prolonged in the subgroup of patients with PANSS MF anxiety/depression $\geq 16$ (Figure 4B). 


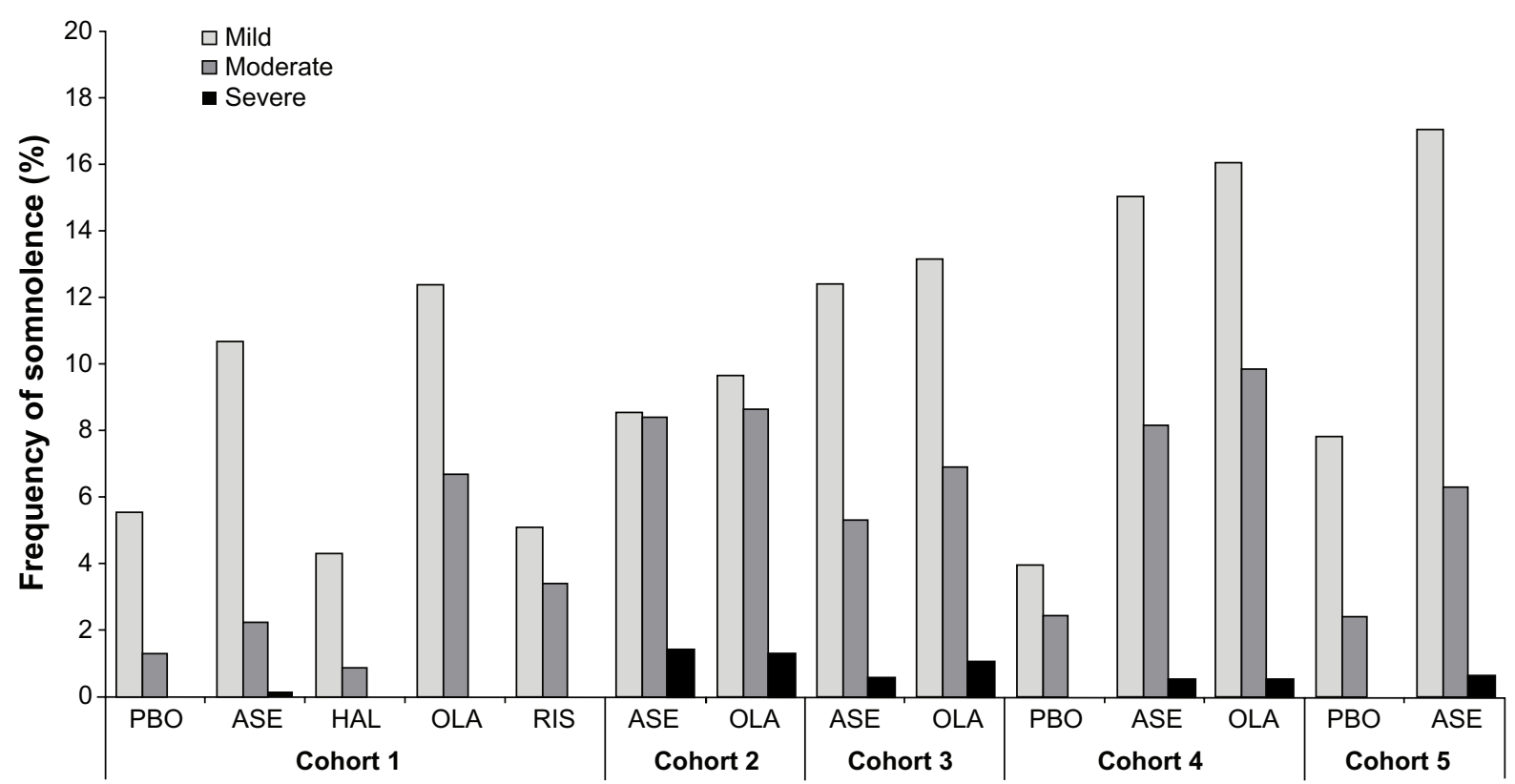

Figure I Frequency of somnolence events by intensity.

Abbreviations: ASE, asenapine; HAL, haloperidol; OLA, olanzapine; PBO, placebo; RIS, risperidone.

\section{Cohort I: short-term schizophrenia rates of sedative/hypnotic use}

In the short-term schizophrenia cohort, $23.5 \%-64.4 \%$ of patients across all treatment groups used sedatives/hypnotics (Table 3 ). Significantly more patients treated with risperidone received sedatives/hypnotics compared with those on placebo, with a difference in rate of 21.8 (95\% CI [8.1, 34.0]). In contrast, significantly fewer patients treated with haloperidol received sedatives/hypnotics than those treated with placebo, with a difference in rate of -19.1 (95\% CI [-27.7, -9.3]). Benzodiazepines were used by $52.2 \%-83.1 \%$ of patients in this cohort during treatment with the study drug. The likelihood of treatment-emergent somnolence occurring with any treatment within 24 hours of receiving hypnotics/benzodiazepines was relatively low (placebo $0.3 \%$, asenapine $2.3 \%$, other antipsychotics $0 \%-1.7 \%$ ).

Similarly, in patients with less severe baseline anxiety (PANSS G2 <4) or symptoms of depression (PANSS G6 $<4$ or PANSS MF $<16$ ), significantly more treated with risperidone but significantly fewer treated with haloperidol received sedatives/hypnotics than those treated with placebo (Table 3). No significant change was observed in the use of sedatives/hypnotics by patients in these groups who were administered asenapine. In contrast, with the exception of those treated with risperidone, significantly fewer patients with severe baseline anxiety (PANSS G2 $\geq 4$ ) treated with other antipsychotics received sedatives/hypnotics than those treated with placebo. In patients with worse baseline anxiety (PANSS G2 $\geq 4$ ), use of benzodiazepines ranged from
$65.6 \%$ to $90.6 \%$ across all groups. In patients with less severe baseline anxiety (PANSS G2 <4), use of benzodiazepines ranged from $43.6 \%$ to $74.1 \%$.

In patients with worse symptoms of depression at baseline (PANSS MF $\geq 16$ ), use of sedatives/hypnotics ranged from $37.5 \%$ to $71.4 \%$. Use of benzodiazepines ranged from $70.4 \%$ to $87.5 \%$ across all groups. In patients with baseline PANSS G6 $\geq 4$, use of sedatives/hypnotics ranged from $40.9 \%$ to $63.2 \%$ and benzodiazepines from $70.1 \%$ to $89.5 \%$ across all treatment groups. In patients with less severe depression at baseline, use of sedatives/hypnotics was $20.4 \%-64.7 \%$ with PANSS MF anxiety/depression $<16$ and $19.4 \%-65.0 \%$ with PANSS G6 $<4$. Use of benzodiazepines was $50.0 \%-82.4 \%$ in patients with baseline PANSS MF anxiety/depression $<16$ and $46.2 \%-80.0 \%$ in those with PANSS G6 $<4$.

\section{Cohort 2: long-term schizophrenia and schizoaffective disorder}

In the cohort with long-term (52-week) schizophrenia and schizoaffective disorder, the incidence of somnolence with asenapine was $18.5 \%$ and $19.6 \%$ with olanzapine, without any significant difference between the two groups. Thirteen patients (1.43\%) treated with asenapine and four (1.29\%) treated with olanzapine experienced severe somnolence (Figure 1). Nine patients (1.0\%) receiving asenapine and one receiving olanzapine $(0.3 \%)$ discontinued as a result of somnolence/sedation. No statistically significant difference was observed for median time to onset of somnolence between 

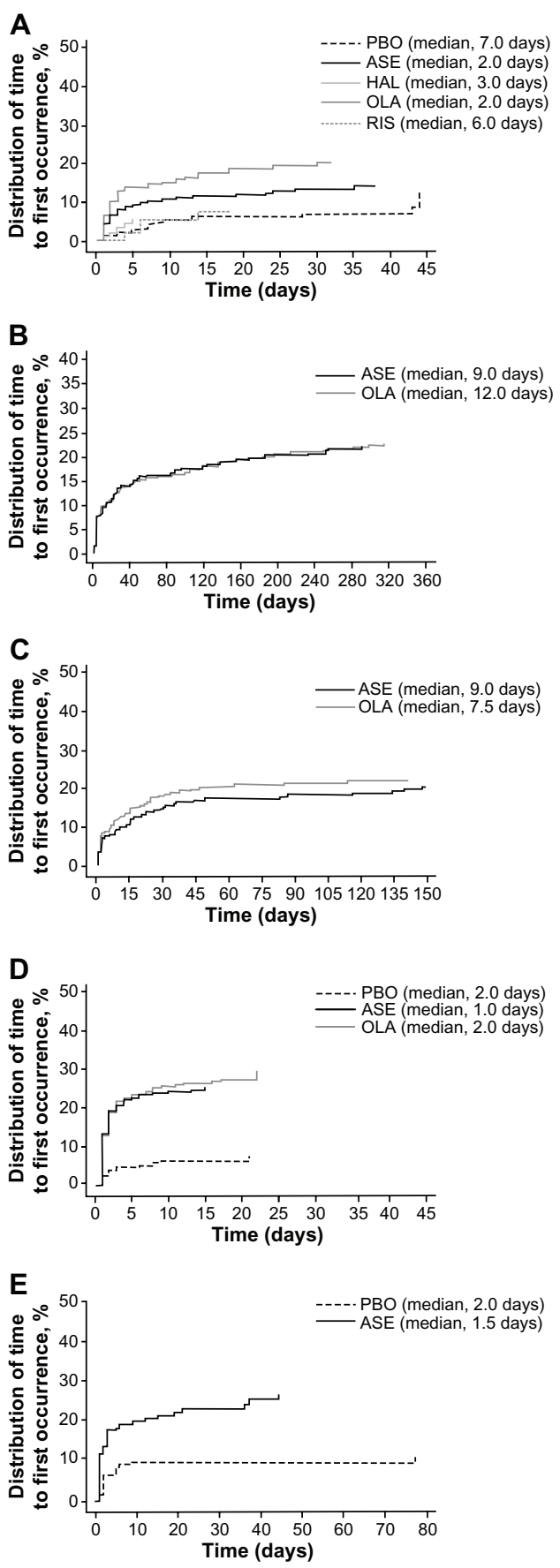

Figure 2 Distribution of time to first occurrence of somnolence in (A) cohort I, (B) cohort 2, (C) cohort 3, (D) cohort 4, and (E) cohort 5.

Abbreviations: ASE, asenapine; HAL, haloperidol; OLA, olanzapine; PBO, placebo; RIS, risperidone.

asenapine and olanzapine ( 9.0 versus 12.0 days, respectively; $P=0.8007$, Figure 2B). The median duration of somnolence with asenapine was similar to that with olanzapine (22.0 versus 21.0 days, respectively, Figure 3A). Of those patients treated with asenapine and olanzapine, $25.8 \%$ and $16.1 \%$, respectively, used sedatives or hypnotics, and $49.3 \%$ and $43.4 \%$ used benzodiazepines. The incidence of somnolence occurring

\section{A}

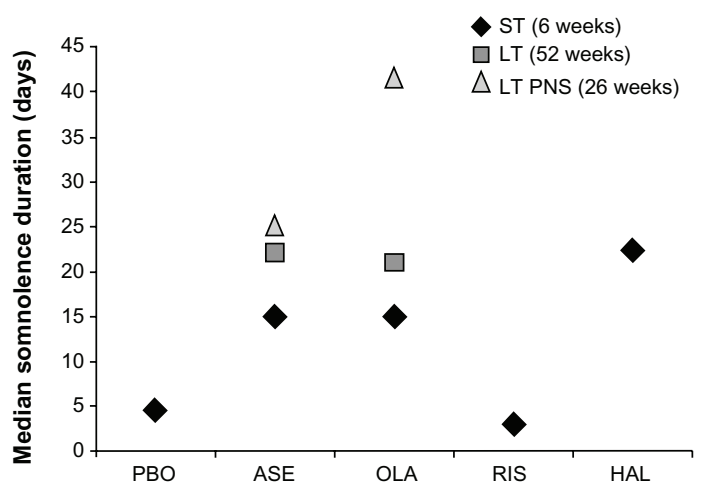

B

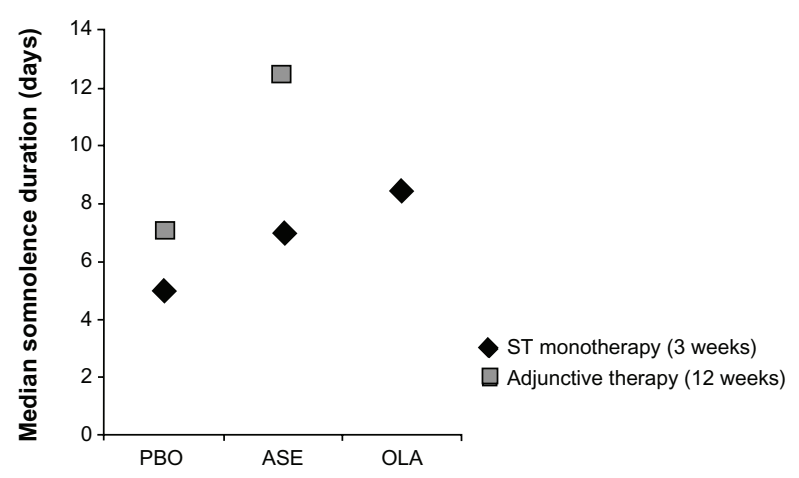

Figure 3 Median duration of somnolence by antipsychotic agent in patients with (A) schizophrenia and (B) bipolar disorder.

Abbreviations: ASE, asenapine; HAL, haloperidol; OLA, olanzapine; PBO, placebo; PNS, persistent negative symptoms; RIS, risperidone; ST, short-term; LT, long-term.

within 24 hours of receiving benzodiazepines was $0.3 \%$ with asenapine and $1.3 \%$ with olanzapine.

\section{Cohort 3: schizophrenia with PNS}

The incidence of somnolence was similar for asenapine (18.4\%) and olanzapine (21.1\%) in the cohort with schizophrenia and PNS. The majority of somnolence events were mild or moderate, with three patients $(0.62 \%)$ treated with asenapine and five patients (1.08\%) treated with olanzapine experiencing severe somnolence (Figure 1). Five patients $(1.0 \%)$ in the asenapine group and three $(0.7 \%)$ in the olanzapine group discontinued as a result of somnolence/sedation. No statistically significant difference in median time to onset of somnolence was observed between asenapine and olanzapine (9.0 days versus 7.5 days, respectively; $P=0.2152$, Figure 2C). Median duration of somnolence (Figure 3A) with asenapine was numerically shorter than with olanzapine (25.0 days versus 41.5 days, respectively).

In the cohort with schizophrenia and PNS, $15.9 \%$ and $11.9 \%$ of patients treated with asenapine and olanzapine, respectively, used sedatives/hypnotics, and $28.3 \%$ and $20.9 \%$ 

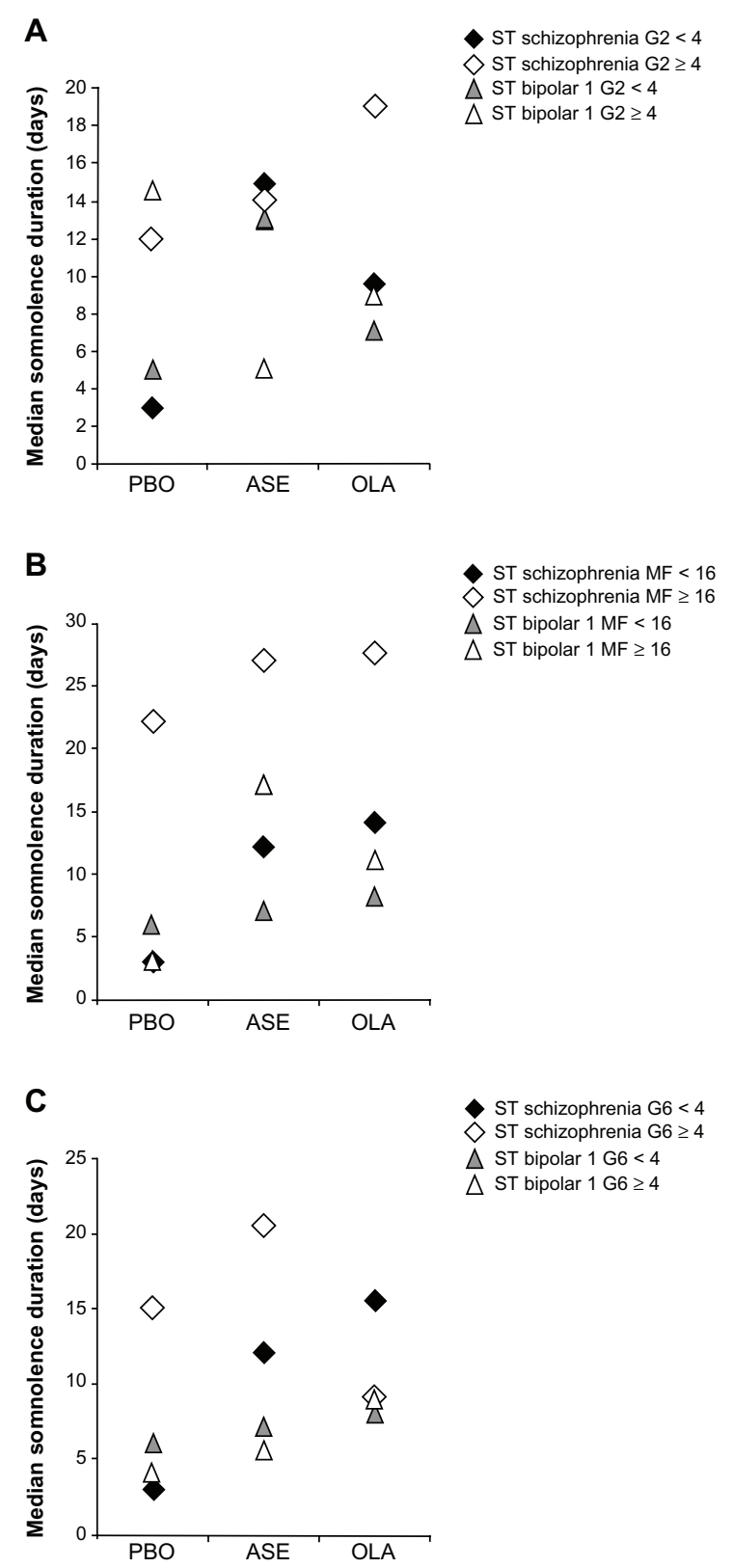

Figure 4 Median duration of somnolence in short-term studies of patients with schizophrenia or bipolar I disorder with baseline (A) anxiety (PANSS G2 $<4$ or $\geq 4$ ), (B) anxiety/depression (PANSS MF anxiety/depression $<16$ or $\geq 16$ ), and (C) depression (PANSS G6 $<4$ or $\geq 4$ ).

Abbreviations: ASE, asenapine; OLA, olanzapine; PBO, placebo; ST, short-term; PANSS, Positive and Negative Syndrome Scale.

used benzodiazepines. The incidence of somnolence within 24 hours of receiving benzodiazepines was low (asenapine $0.4 \%$, olanzapine $0.2 \%$ ).

\section{Bipolar I mania}

\section{Cohort 4: risk of somnolence with 3-week} monotherapy

In the 3-week bipolar I monotherapy cohort, the incidence of somnolence in the asenapine and olanzapine groups was significantly higher than that in the placebo group, with a NNH of $6(95 \%$ CI $[4,9])$ and $5.0(95 \%$ CI $[4,7])$, respectively (Table 4$)$. No patient in the placebo group, one $(0.3 \%)$ in the asenapine group, and two $(0.5 \%)$ in the olanzapine group discontinued because of somnolence/ sedation. In both active arms, patients with lower (PANSS G2 $<4$ ) or higher (PANSS G2 $\geq 4$ ) baseline anxiety had significantly higher rates of somnolence than in the placebo group, although the NNH was smaller in those with worse anxiety. Further, patients with less or more severe symptoms of depression (PANSS G6 $<$ or $\geq 4$ ) had significantly higher rates of somnolence with both active arms than with placebo. Severe somnolence was experienced by two patients $(0.53 \%)$ treated with asenapine, two patients $(0.51 \%)$ treated with olanzapine, and none with placebo (Figure 1).

No statistically significant difference in median time to onset of somnolence (Figure 2D) was observed with asenapine ( 1.0 days; $P=0.0780$ ) or olanzapine (2.0 days; $P=0.4375$ ), compared with placebo (2.0 days). The median duration of somnolence (Figure 3B) with asenapine (7 days) was longer than with placebo (5 days), but shorter than with olanzapine ( 8.5 days). A numerical decrease in duration of somnolence was observed in patients with PANSS G2 $\geq 4$ receiving asenapine (Figure 4A). No further clear differences were observed in duration of somnolence for the patient subgroups with either lower or elevated anxiety or depression, as measured by PANSS MF anxiety/depression and PANSS G6 depression scores (Figures 4B and 4C). In addition, no clear differences were observed in median duration of somnolence in patients with fewer or more symptoms of depression as measured by MADRS $<20$ or $\geq 20$ (placebo 7 versus 4 days, asenapine 8 versus 4 days, olanzapine 8 versus 11 days) or CGI-BD-S $<4$ or $\geq 4$ (placebo, 7 days versus 3 days; asenapine, 10.5 days versus 5 days; olanzapine, 7 days versus 14 days).

\section{Cohort 4: rate of sedative/hypnotic use with 3-week monotherapy}

In the 3-week bipolar I monotherapy cohort, 35.1\%-37.0\% of patients across all treatment groups used sedatives/ hypnotics, and $53.8 \%-63.6 \%$ used benzodiazepines. There were no significant differences between patients treated with antipsychotics and placebo with regard to rates of using sedatives/hypnotics (Table 5). However, patients with less severe baseline anxiety or depression tended to use fewer sedatives/hypnotics across the three treatment arms compared with those with worse anxiety and depression. 
Table 3 Sedative/hypnotic use in short-term schizophrenia cohort

\begin{tabular}{|c|c|c|c|}
\hline & Treatment & $\begin{array}{l}\text { Sedative/hypnotic use } \\
\text { n/N (\%) }\end{array}$ & $\begin{array}{l}\text { Difference between active } \\
\text { treatment and placebo } \%(95 \% \mathrm{Cl})\end{array}$ \\
\hline \multirow[t]{5}{*}{ Full sample } & Asenapine 5-10 mg twice daily & $208 / 572(36.4)$ & $-6.2(-12.6,0.1)$ \\
\hline & Risperidone 3 mg twice daily & $38 / 59(64.4)$ & $21.8(8.1,34.0)$ \\
\hline & Olanzapine $5-20 \mathrm{mg}$ once daily & $82 / 194(42.3)$ & $-0.3(-8.8,8.3)$ \\
\hline & Haloperidol 4 mg twice daily & $27 / 115(23.5)$ & $-19.1(-27.7,-9.3)$ \\
\hline & Placebo & $161 / 378(42.6)$ & - \\
\hline \multirow[t]{5}{*}{$\mathrm{G} 2<4$} & Asenapine 5-10 mg twice daily & $|00 / 33|(30.2)$ & $-2.8(-10.9,5.0)$ \\
\hline & Risperidone 3 mg twice daily & $17 / 27(63.0)$ & $29.9(10.1,46.8)$ \\
\hline & Olanzapine $5-20 \mathrm{mg}$ once daily & $42 / 100(42.0)$ & $9.0(-2.3,20.5)$ \\
\hline & Haloperidol 4 mg twice daily & I4/78 (I8.0) & $-15.1(-24.8,-3.6)$ \\
\hline & Placebo & $72 / 218(33.0)$ & - \\
\hline \multirow[t]{5}{*}{$\mathrm{G} 2 \geq 4$} & Asenapine 5-10 mg twice daily & $|08 / 24|(44.8)$ & $-10.8(-20.6,-0.8)$ \\
\hline & Risperidone $3 \mathrm{mg}$ twice daily & $2 \mathrm{I} / 32(65.6)$ & $10.0(-9.0,26.3)$ \\
\hline & Olanzapine $5-20 \mathrm{mg}$ once daily & $40 / 94(42.6)$ & $-13.1(-25.4,-0.3)$ \\
\hline & Haloperidol 4 mg twice daily & 13/37 (35.1) & $-20.5(-36.1,-2.6)$ \\
\hline & Placebo & $89 / 160(55.6)$ & - \\
\hline \multirow[t]{5}{*}{$\mathrm{MF}<16$} & Asenapine $5-10 \mathrm{mg}$ twice daily & |78/5 I 8 (34.4) & $-6.1(-12.7,0.6)$ \\
\hline & Risperidone 3 mg twice daily & $33 / 51(64.7)$ & $24.3(9.6,37.2)$ \\
\hline & Olanzapine $5-20 \mathrm{mg}$ once daily & $76 / 178(42.7)$ & $2.3(-6.6,11.3)$ \\
\hline & Haloperidol 4 mg twice daily & $22 / 108(20.4)$ & $-20.0(-28.6,-10.0)$ \\
\hline & Placebo & I37/339 (40.4) & - \\
\hline \multirow[t]{5}{*}{$M F \geq 16$} & Asenapine $5-10 \mathrm{mg}$ twice daily & $30 / 54(55.6)$ & $-6.0(-25.5,14.4)$ \\
\hline & Risperidone 3 mg twice daily & $5 / 8(62.5)$ & $1.0(-35.1,3 \mid .0)$ \\
\hline & Olanzapine $5-20 \mathrm{mg}$ once daily & 6/16 (37.5) & $-24.0(-48.9,5.0)$ \\
\hline & Haloperidol 4 mg twice daily & $5 / 7(7 \mid .4)$ & $9.9(-29.4,37.5)$ \\
\hline & Placebo & $24 / 39(61.5)$ & - \\
\hline \multirow[t]{5}{*}{$\mathrm{G} 6<4$} & Asenapine 5-10 mg twice daily & $140 / 428(32.7)$ & $-4.7(-12.0,2.5)$ \\
\hline & Risperidone $3 \mathrm{mg}$ twice daily & $26 / 40(65.0)$ & $27.6(11.0,41.9)$ \\
\hline & Olanzapine 5-20 mg once daily & $56 / 145(38.6)$ & $1.2(-8.4,1 \mathrm{I} . \mathrm{I})$ \\
\hline & Haloperidol 4 mg twice daily & 18/93 (19.4) & $-18.1(-27.2,-7.4)$ \\
\hline & Placebo & $101 / 270(37.4)$ & - \\
\hline \multirow[t]{5}{*}{$\mathrm{G} 6 \geq 4$} & Asenapine 5-10 mg twice daily & $68 / 144(47.2)$ & $-8.3(-20.5,4.2)$ \\
\hline & Risperidone 3 mg twice daily & $12 / 19(63.2)$ & $7.6(-16.6,28.2)$ \\
\hline & Olanzapine $5-20 \mathrm{mg}$ once daily & $26 / 49(53.1)$ & $-2.5(-19.1,14.0)$ \\
\hline & Haloperidol 4 mg twice daily & $9 / 22(40.9)$ & $-14.6(-35.0,8.0)$ \\
\hline & Placebo & $60 / 108(55.6)$ & - \\
\hline
\end{tabular}

Abbreviations: $\mathrm{Cl}$, confidence interval; $\mathrm{N}$, total number of patients in treatment arm; $\mathrm{n}$, number of patients with treatment-emergent somnolence; \%, percentage of patients in treatment arm with treatment-emergent somnolence; G2, PANSS G2 (anxiety); MF, PANSS MF anxiety/depression; G6, PANSS G6 (depression); PANSS, Positive and Negative Syndrome Scale.

\section{Cohort 5: I 2-week adjunctive therapy in bipolar I mania}

In the bipolar I adjunctive therapy cohort, the rate of somnolence was significantly higher with asenapine $(24.1 \%)$ than with placebo $(10.2 \%)$, with a NNH of $7(95 \%$ CI $[5,18]$, Table 4$)$. One patient in each group $(0.6 \%)$ discontinued due to somnolence/sedation. One patient treated with asenapine and none treated with placebo experienced severe somnolence (Figure 1). Compared with placebo, no statistically significant difference in median time to onset of somnolence was observed with asenapine (2.0 days and 1.5 days, respectively; $P=0.6697$, Figure $2 \mathrm{E}$ ). The median duration of somnolence with asenapine was longer than with placebo (12.5 days versus 7.0 days, Figure 3B). During the study period, $41.6 \%$ with asenapine and $43.0 \%$ with placebo used sedatives/hypnotics, and 54.8 and $53.2 \%$ used benzodiazepines. The incidence of somnolence within 24 hours of receiving benzodiazepines was low (placebo, 1.8\%; asenapine, $4.4 \%)$.

\section{Discussion}

Somnolence and sedation are common side effects of antipsychotic medications prescribed for schizophrenia or $\mathrm{BPD},{ }^{2}$ and may result in unintentional injury, ${ }^{18}$ impaired functioning, and noncompliance with treatment. Asenapine has been shown to be associated with somnolence/sedation in 
Table 4 Incidence of somnolence in short-term bipolar I monotherapy and adjunctive cohorts

\begin{tabular}{|c|c|c|c|c|}
\hline & Treatment & $\begin{array}{l}\text { Incidence } \\
\mathrm{n} / \mathrm{N} \text { (\%) }\end{array}$ & $\begin{array}{l}\text { Difference between active treatment } \\
\text { and placebo } \%(95 \% \mathrm{Cl})\end{array}$ & $\begin{array}{l}\text { NNH mean } \\
(95 \% \mathrm{Cl})\end{array}$ \\
\hline \multicolumn{5}{|c|}{ Short-term bipolar I monotherapy } \\
\hline \multirow[t]{3}{*}{ Full sample } & Asenapine $5-10 \mathrm{mg}$ twice daily & $90 / 379(23.8)$ & $17.34(\mid 1.64,22.72)$ & $6(4,9)$ \\
\hline & Olanzapine $5-20 \mathrm{mg}$ once daily & $104 / 394(26.4)$ & $19.99(14.23,25.40)$ & $5(4,7)$ \\
\hline & Placebo & $13 / 203(6.4)$ & - & - \\
\hline \multirow[t]{3}{*}{$\mathrm{G} 2<4$} & Asenapine 5-10 mg twice daily & $50 / 249(20.1)$ & $13.16(6.03,19.65)$ & $8(5,17)$ \\
\hline & Olanzapine $5-20 \mathrm{mg}$ once daily & $58 / 254(22.8)$ & $|5.9|(8.66,22.53)$ & $6(4,12)$ \\
\hline & Placebo & $9 / 130(6.9)$ & - & - \\
\hline \multirow[t]{3}{*}{$\mathrm{G} 2 \geq 4$} & Asenapine $5-10 \mathrm{mg}$ twice daily & $40 / 130(30.8)$ & $25.29(15.06,34.68)$ & $4(3,7)$ \\
\hline & Olanzapine $5-20 \mathrm{mg}$ once daily & $46 / 140(32.9)$ & $27.38(17.20,36.55)$ & $4(3,6)$ \\
\hline & Placebo & $4 / 73(5.5)$ & - & - \\
\hline \multirow[t]{3}{*}{$M F<16$} & Asenapine $5-10 \mathrm{mg}$ twice daily & $81 / 347(23.3)$ & I7.66 (II.74, 23.20) & $6(4,9)$ \\
\hline & Olanzapine $5-20 \mathrm{mg}$ once daily & $95 / 361$ (26.3) & $20.63(14.63,26.22)$ & $5(4,7)$ \\
\hline & Placebo & $10 / 176(5.7)$ & - & - \\
\hline \multirow[t]{3}{*}{$M F \geq 16$} & Asenapine 5-10 mg twice daily & $9 / 32(28.1)$ & $|7.0|(-4.16,36.74)$ & ns \\
\hline & Olanzapine $5-20 \mathrm{mg}$ once daily & 9/33 (27.3) & $16.16(-4.82,35.58)$ & ns \\
\hline & Placebo & $3 / 27(11.1)$ & - & - \\
\hline \multirow[t]{3}{*}{$\mathrm{G} 6<4$} & Asenapine 5-10 mg twice daily & $66 / 313(21.1)$ & I5.32 (9.|I, 21.04) & $7(5,11)$ \\
\hline & Olanzapine $5-20 \mathrm{mg}$ once daily & $84 / 329(25.5)$ & $19.76(\mid 3.4 I, 25.6 I)$ & $5(4,8)$ \\
\hline & Placebo & $9 / 156(5.8)$ & - & - \\
\hline \multirow[t]{3}{*}{$\mathrm{G} 6 \geq 4$} & Asenapine $5-10 \mathrm{mg}$ twice daily & $24 / 66(36.4)$ & 27.85 (I2.72, 4I.59) & $4(2,8)$ \\
\hline & Olanzapine $5-20 \mathrm{mg}$ once daily & $20 / 65(30.8)$ & $22.26(7.49,35.93)$ & $5(3,13)$ \\
\hline & Placebo & $4 / 47(8.5)$ & - & - \\
\hline \multirow[t]{3}{*}{ MADRS $<20$} & Asenapine $5-10 \mathrm{mg}$ twice daily & $77 / 334(23.1)$ & $17.73(\mid I .77,23.3 I)$ & $6(4,9)$ \\
\hline & Olanzapine $5-20 \mathrm{mg}$ once daily & $87 / 34 \mid(25.5)$ & $20.19(14.14,25.84)$ & $5(4,7)$ \\
\hline & Placebo & $9 / 169(5.3)$ & - & - \\
\hline \multirow[t]{3}{*}{ MADRS $\geq 20$} & Asenapine 5-10 mg twice daily & 13/45 (28.9) & $17.12(-1.42,34.04)$ & ns \\
\hline & Olanzapine $5-20 \mathrm{mg}$ once daily & $17 / 53(32.1)$ & $20.31(1.96,36.28)$ & $5(3,5 I)$ \\
\hline & Placebo & $4 / 34(11.8)$ & - & - \\
\hline \multirow[t]{3}{*}{ CGI-BD-S $<4$} & Asenapine $5-10 \mathrm{mg}$ twice daily & $72 / 320(22.5)$ & $15.83(9.52,21.68)$ & $6(5,11)$ \\
\hline & Olanzapine $5-20 \mathrm{mg}$ once daily & $85 / 321(26.5)$ & I9.8I (I3.32, 25.84) & $5(4,8)$ \\
\hline & Placebo & $1 \mathrm{I} / 165(6.7)$ & - & - \\
\hline \multirow[t]{3}{*}{ CGI-BD-S $\geq 4$} & Asenapine $5-10 \mathrm{mg}$ twice daily & $18 / 59(30.5)$ & $25.25(10.09,39.06)$ & $4(3,10)$ \\
\hline & Olanzapine $5-20 \mathrm{mg}$ once daily & $19 / 73(26.0)$ & $20.76(6.43,33.06)$ & $5(3,16)$ \\
\hline & Placebo & $2 / 38(5.3)$ & - & - \\
\hline \multicolumn{5}{|c|}{ Bipolar I adjunctive } \\
\hline \multirow[t]{2}{*}{ Full sample } & Asenapine $5-10 \mathrm{mg}$ twice daily & $38 / 158(24.1)$ & $|3.8|(5.73,22.11)$ & $7(5,18)$ \\
\hline & Placebo & $17 / 166(10.2)$ & - & - \\
\hline
\end{tabular}

Abbreviations: CGI-BD-S, Clinical Global Impression for Bipolar Disorder Severity; MADRS, Montgomery-Åsberg Depression Rating Scale; MF, PANSS MF anxiety/ depression; G2, PANSS G2 (anxiety); G6, PANSS G6 (depression); N, total number of patients in treatment arm; n, number of patients with treatment-emergent somnolence; $\%$, percentage of patients in treatment arm with treatment-emergent somnolence; $\mathrm{NNH}$, number needed to treat to result in an adverse event of somnolence (harm); ns, not statistically significant; PANSS, Positive and Negative Syndrome Scale.

patients with schizophrenia and bipolar I disorder. ${ }^{11,14}$ However, to the authors' knowledge, this is the first report to examine treatment-emergent somnolence with asenapine and other antipsychotics in the treatment of schizophrenia and BPD systematically using incidence rates, time to onset, duration, severity of somnolence, and use of sedatives/hypnotics. This may also be the first study using the PANSS and MADRS to explore the relationship between treatment-emergent somnolence and the baseline severity of anxiety and depression.

The results of this study confirm that somnolence/sedation occurs with asenapine in a limited number (13\%-24\%) of patients with schizophrenia or BPD early in the course of treatment, when it is of limited duration and generally of mild to moderate intensity. More importantly, treatmentemergent somnolence with asenapine was not associated with an increased risk of discontinuation of treatment, which is consistent with findings for other antipsychotics in the treatment of schizophrenia and BPD. ${ }^{2}$ Our results for treatment-emergent somnolence with olanzapine, risperidone, and haloperidol (Table 1) were also consistent with those of a previous study by Gao et $\mathrm{al}^{2}{ }^{2}$ in which olanzapine, but not risperidone or haloperidol, had an increased risk for somnolence relative to placebo in the treatment of schizophrenia. 
Table 5 Sedative/hypnotic use in bipolar mania studies

\begin{tabular}{|c|c|c|c|}
\hline & Treatment & $\begin{array}{l}\text { Sedative/hypnotic use } \\
\text { n/N (\%) }\end{array}$ & $\begin{array}{l}\text { Difference between active } \\
\text { treatment and placebo } \%(95 \% \mathrm{Cl})\end{array}$ \\
\hline \multicolumn{4}{|c|}{ Short-term bipolar I monotherapy } \\
\hline \multirow[t]{3}{*}{ Full sample } & Asenapine 5-10 mg twice daily & $133 / 379(35.1)$ & $-1.9(-10.1,6.2)$ \\
\hline & Olanzapine $5-20 \mathrm{mg}$ once daily & $142 / 394(36.0)$ & $-0.9(-9.2,7.1)$ \\
\hline & Placebo & $75 / 203(37.0)$ & - \\
\hline \multirow[t]{3}{*}{$\mathrm{G} 2<4$} & Asenapine 5-10 mg twice daily & $74 / 249(29.7)$ & $-2.6(-12.6,7.0)$ \\
\hline & Olanzapine $5-20 \mathrm{mg}$ once daily & $76 / 254(29.9)$ & $-2.4(-12.4,7.2)$ \\
\hline & Placebo & $42 / 130(32.3)$ & - \\
\hline \multirow[t]{3}{*}{$\mathrm{G} 2 \geq 4$} & Asenapine 5-10 mg twice daily & $59 / 130(45.4)$ & $0.2(-14.1,14.2)$ \\
\hline & Olanzapine $5-20 \mathrm{mg}$ once daily & $66 / 140(47.1)$ & $1.9(-12.1,15.8)$ \\
\hline & Placebo & $33 / 73(45.2)$ & - \\
\hline \multirow[t]{3}{*}{$M F<16$} & Asenapine 5-10 mg twice daily & I I 8/347 (34.0) & $-0.1(-8.8,8.3)$ \\
\hline & Olanzapine $5-20 \mathrm{mg}$ once daily & $|27 / 36|(35.2)$ & I.I $(-7.6,9.5)$ \\
\hline & Placebo & $60 / 176(34.1)$ & - \\
\hline \multirow[t]{3}{*}{$M F \geq 16$} & Asenapine 5-10 mg twice daily & I5/32 (46.9) & $-8.7(-33,16.8)$ \\
\hline & Olanzapine $5-20 \mathrm{mg}$ once daily & I5/33 (45.5) & $-10.1(-34.2,15.2)$ \\
\hline & Placebo & $15 / 27(55.6)$ & - \\
\hline \multirow[t]{3}{*}{$\mathrm{G} 6<4$} & Asenapine 5-10 mg twice daily & $101 / 313(32.3)$ & $0.9(-8.3,9.6)$ \\
\hline & Olanzapine $5-20 \mathrm{mg}$ once daily & $104 / 329(31.6)$ & $0.2(-8.9,8.8)$ \\
\hline & Placebo & $49 / 156(31.4)$ & - \\
\hline \multirow[t]{3}{*}{$\mathrm{G} 6 \geq 4$} & Asenapine 5-10 mg twice daily & $32 / 66(48.5)$ & $-6.8(-24.9,11.8)$ \\
\hline & Olanzapine $5-20 \mathrm{mg}$ once daily & $38 / 65(58.5)$ & $3.1(-15.2,21.5)$ \\
\hline & Placebo & $26 / 47(55.3)$ & - \\
\hline \multirow[t]{3}{*}{ MADRS $<20$} & Asenapine $5-10 \mathrm{mg}$ twice daily & II3/334 (33.8) & $0.1(-8.8,8.7)$ \\
\hline & Olanzapine $5-20 \mathrm{mg}$ once daily & $|I 6 / 34|(34.0)$ & $0.3(-8.6,8.8)$ \\
\hline & Placebo & $57 / 169(33.7)$ & - \\
\hline \multirow[t]{3}{*}{ MADRS $\geq 20$} & Asenapine 5-10 mg twice daily & $20 / 45(44.4)$ & $-8.5(-29.9,13.6)$ \\
\hline & Olanzapine $5-20 \mathrm{mg}$ once daily & $26 / 53(49.1)$ & $-3.9(-24.8,17.4)$ \\
\hline & Placebo & $18 / 34(52.9)$ & - \\
\hline \multirow[t]{3}{*}{ CGI-BD-S $<4$} & Asenapine 5-10 mg twice daily & $100 / 320(31.3)$ & $-1.5(-10.4,7.1)$ \\
\hline & Olanzapine $5-20 \mathrm{mg}$ once daily & $103 / 321(32.1)$ & $-0.6(-9.6,7.9)$ \\
\hline & Placebo & $54 / 165(32.7)$ & - \\
\hline \multirow[t]{3}{*}{ CGI-BD-S $\geq 4$} & Asenapine 5-10 mg twice daily & $33 / 59(55.9)$ & $0.7(-19.1,20.7)$ \\
\hline & Olanzapine $5-20 \mathrm{mg}$ once daily & $39 / 73(53.4)$ & $-1.8(-20.7,17.6)$ \\
\hline & Placebo & $21 / 38(55.3)$ & - \\
\hline \multicolumn{4}{|c|}{ Bipolar I adjunctive } \\
\hline \multirow[t]{2}{*}{ Full sample } & Asenapine 5-10 mg twice daily & 68/I58 (43.0) & $1.5(-9.3,12.2)$ \\
\hline & Placebo & $69 / 166(41.6)$ & - \\
\hline
\end{tabular}

Abbreviations: CGI-BD-S, Clinical Global Impression for bipolar disorder; MADRS, Montgomery-Åsberg Depression Rating Scale; MF, PANSS MF anxiety/depression; G2, PANSS G2 (anxiety); G6, PANSS G6 (depression); PANSS, Positive and Negative Syndrome Scale.

Treatment-emergent somnolence associated with asenapine was more similar to that with olanzapine than with other antipsychotics with respect to time to onset and duration of somnolence (Figures 1 and 2). However, the risk of somnolence with asenapine was less than that for olanzapine, as reflected by an $\mathrm{NNH}$ of 16 for asenapine and 8 for olanzapine (Table 2). Both olanzapine and asenapine bind to a broad range of receptor subtypes, including $\mathrm{H}_{1}$ receptors. ${ }^{3,19} \mathrm{H}_{1}$ antagonists are well known to be associated with sedation, ${ }^{20}$ and the difference in risk of somnolence could be a result of the greater affinity of olanzapine for $\mathrm{H}_{1}$ receptors compared with asenapine. However, the risk of somnolence in bipolar I mania was similar (NNH 6 for asenapine; 5 for olanzapine).
An increased risk of somnolence in patients with bipolar mania compared with those having schizophrenia has been reported previously for other antipsychotics, including risperidone, quetiapine, aripiprazole, and ziprasidone, but not for olanzapine. ${ }^{2}$ In the post hoc analyses described here, the greater sensitivity to somnolence associated with asenapine of patients with bipolar mania compared to those with schizophrenia (NNH 16 for schizophrenia; 6 for bipolar mania) was similar to that previously reported for aripiprazole (NNH 14 for schizophrenia; 8 for bipolar mania).

Hypnotics, sedatives, and benzodiazepines were widely used throughout the studies and it is possible that a degree of somnolence may be associated with the use of these 
sedating drugs. However, the incidence of somnolence did not appear to be related directly to the use of hypnotics or benzodiazepines, because the risk of developing somnolence within 24 hours of administering these drugs was very low. This suggests that the increased occurrence of somnolence with asenapine and olanzapine may be a "true" effect of these two medications. In addition, the finding that more patients treated with risperidone used sedatives/hypnotics seems consistent with the less "sedating" pharmacological profile of this agent (Table 3 ).

It is well known that patients with schizophrenia or BPD often have inadequate sleep and sleep disturbances. ${ }^{21,22}$ Under these circumstances, antipsychotic-related somnolence may actually prove beneficial in alleviating these symptoms as well as agitation. However, the finding that patients with higher baseline anxiety were more likely to experience somnolence suggests that clinicians may need to focus attention on this group of patients to prevent discontinuation because of somnolence/sedation. Further, patients with schizophrenia and worse baseline anxiety $(\mathrm{G} 2 \geq 4)$ were less likely to receive sedatives/hypnotics with each of the antipsychotics relative to those on placebo. These data further support that the treatment-emergent somnolence was not related to use of sedatives/hypnotics. Importantly, these data expand upon the previous finding by Gao et al that patients with generalized anxiety disorder were more sensitive to antipsychotics than patients with schizophrenia, BPD, or major depressive disorder. $^{23}$

Patients with schizophrenia or BPD with less severe depression at baseline tended to be at higher risk of treatmentemergent somnolence with asenapine or olanzapine relative to placebo than those with more severe depression at baseline. The findings of this post hoc analysis will need to be confirmed by further research, in view of the variation in size of the underlying databases for each of the compounds studied.

\section{Limitations}

The results of this study are limited by the original study designs, including the methods used to collect data and intervals between study visits. For instance, visit schedules for cohort 1 were at day 4 and weeks $1,2,3,4,5$, and 6 whereas those for cohort 2 were at weeks 1, 2, 3, 4, 6 , and 8 , and then monthly. The median time to onset of somnolence with asenapine and olanzapine in cohort 1 was 2 days for both drugs, whereas the median time to onset of somnolence with asenapine and olanzapine in cohort 2 was 9 days and 12 days, respectively. The difference in time to onset of somnolence between cohorts 1 and 2 could be due to lack of assessment at day 4 in cohort 2 or different dosing schedules being used in cohorts 1 and 2. Given that the median time to onset of somnolence with asenapine and olanzapine in cohort 2 was longer than 7 days, it is more likely that the longer median time to onset in cohort 2 was due to the flexible-dosing schedule used. In cohort 1 , the dosing schedule was fixed, which might be a reason for the shorter median time to onset of somnolence. The flexible-dosing schedule in cohort 2 is similar to that used in routine clinical care. Therefore, the findings from cohort 2 might be more clinically relevant.

These results could be confounded further by uncontrolled factors related to sleep-wake cycles in the original studies, such as consumption of caffeine, alcohol, nicotine, prescribed and nonprescribed drugs, quality and quantity of night-time sleep, medical conditions such as thyroid disease, and other sleep disturbances. Further, we counted the frequency of somnolence as an adverse event using MedDRA preferred terms, including somnolence, sedation, and hypersomnia. If there was more than one event reported, that with maximal severity was chosen. Clearly, this method not only ignored the severity of somnolence, but also may have obscured its prevalence.

\section{Conclusion}

The findings of this post hoc analysis confirm that treatmentemergent somnolence can occur in patients with schizophrenia or BPD receiving treatment with asenapine. In the cohort of patients with short-term schizophrenia, the pattern of time to onset and duration of somnolence with asenapine was similar to those for olanzapine and haloperidol compared with risperidone, but only asenapine and olanzapine had significantly higher rates of somnolence compared with placebo. In the cohort with long-term schizophrenia or BPD, the patterns of time to onset, duration, and incidence of somnolence for asenapine and olanzapine were similar. This study confirms our previous finding that patients with bipolar mania are more sensitive than those with schizophrenia to the sedative effects of antipsychotics. Our data suggest that although olanzapine has the highest rate of somnolence, clinicians should be aware that somnolence can occur with asenapine when used in the treatment of patients with schizophrenia or BPD. A lower dose or slower titration may be necessary in patients with BPD. On the other hand, the early onset and limited duration of treatment-emergent somnolence associated with asenapine may be useful in certain clinical situations, such as agitation and/or insomnia. 


\section{Disclosure}

KG was a consultant for Schering-Plough, has received grant support from AstraZeneca and NARSAD, and was on an advisory board for Schering-Plough and a speaker's bureau for Pfizer. MM and AS are shareholders and/or full-time employees of Merck. PC and JZ were full time employees of Merck at the time of the research. Medical writing assistance was provided by Karen Pemberton PhD of PPSI (a PAREXEL company) and was funded by Merck.

\section{References}

1. Meyer RE, Salzman C, Youngstrom EA, et al. Suicidality and risk of suicide - definition, drug safety concerns, and a necessary target for drug development: a consensus statement. J Clin Psychiatry. 2010;71(8): $\mathrm{e} 1-\mathrm{e} 21$.

2. Gao K, Ganocy SJ, Gajwani P, Muzina DJ, Kemp DE, Calabrese JR. A review of sensitivity and tolerability of antipsychotics in patients with bipolar disorder or schizophrenia: focus on somnolence. J Clin Psychiatry. 2008;69(2):302-309.

3. Shahid M, Walker GB, Zorn SH, Wong EH. Asenapine: a novel psychopharmacologic agent with a unique human receptor signature. J Psychopharmacol. 2009;23(1):65-73.

4. Gerrits MG, de Greef R, Dogterom P, Peeters PA. Valproate reduces the glucuronidation of asenapine without affecting asenapine plasma concentrations. J Clin Pharmacol. 2012;52(5):757-765.

5. Kane JM, Cohen M, Zhao J, Alphs L, Panagides J. Efficacy and safety of asenapine in a placebo- and haloperidol-controlled trial in patients with acute exacerbation of schizophrenia. J Clin Psychopharmacol. 2010;30(2):106-115.

6. Kane JM, Mackle M, Snow-Adami L, Zhao J, Szegedi A, Panagides J. A randomized placebo-controlled trial of asenapine for the prevention of relapse of schizophrenia after long-term treatment. J Clin Psychiatry. 2011;72(3):349-355.

7. Potkin SG, Cohen M, Panagides J. Efficacy and tolerability of asenapine in acute schizophrenia: a placebo- and risperidone-controlled trial. J Clin Psychiatry. 2007;68(10):1492-1500.

8. Schoemaker J, Naber D, Vrijland P, Panagides J, Emsley R. Long-term assessment of asenapine versus olanzapine in patients with schizophrenia or schizoaffective disorder. Pharmacopsychiatry. 2010;43(4): 138-146.

9. McIntyre RS, Cohen M, Zhao J, Alphs L, Macek TA, Panagides J. A 3-week, randomized, placebo-controlled trial of asenapine in the treatment of acute mania in bipolar mania and mixed states. Bipolar Disord. 2009;11(7):673-686.
10. McIntyre RS, Cohen M, Zhao J, Alphs L, Macek TA, Panagides J. Asenapine versus olanzapine in acute mania: a double-blind extension study. Bipolar Disord. 2009;11(8):815-826.

11. McIntyre RS, Cohen M, Zhao J, Alphs L, Macek TA, Panagides J. Asenapine for long-term treatment of bipolar disorder: a double-blind 40-week extension study. J Affect Disord. 2010;126(3):358-365.

12. McIntyre RS, Cohen M, Zhao J, Alphs L, Macek TA, Panagides J. Asenapine in the treatment of acute mania in bipolar I disorder: a randomized, double-blind, placebo-controlled trial. J Affect Disord. 2010; 122(1-2):27-38

13. Szegedi A, Calabrese JR, Stet L, Mackle M, Zhao J, Panagides J. Asenapine as adjunctive treatment for acute mania associated with bipolar disorder: results of a 12 -week core study and 40 -week extension. J Clin Psychopharmacol. 2012;32(1):46-55.

14. Citrome L. Asenapine for schizophrenia and bipolar disorder: a review of the efficacy and safety profile for this newly approved sublingually absorbed second-generation antipsychotic. Int J Clin Pract. 2009; 63(12):1762-1784.

15. Buchanan RW, Panagides J, Zhao J, et al. Asenapine versus olanzapine in people with persistent negative symptoms of schizophrenia. J Clin Psychopharmacol. 2012;32(1):36-45.

16. Szegedi A, Verweij P, van Duijnhoven W, Mackle M, Cazorla P, Fennema $\mathrm{H}$. Meta-analyses of the efficacy of asenapine for acute schizophrenia: comparisons with placebo and other antipsychotics. J Clin Psychiatry. 2012;73(12):1533-1540.

17. Miettinen O, Nurminen M. Comparative analysis of two rates. Stat Med. 1985;4(2):213-226.

18. Said Q, Gutterman EM, Kim MS, Firth SD, Whitehead R, Brixner D. Somnolence effects of antipsychotic medications and the risk of unintentional injury. Pharmacoepidemiol Drug Saf. 2008;17(4): 354-364.

19. Bymaster FP, Calligaro DO, Falcone JF, et al. Radioreceptor binding profile of the atypical antipsychotic olanzapine. Neuropsychopharmacology. 1996;14(2):87-96.

20. Nicholson AN, Pascoe PA, Turner C, et al. Sedation and histamine H1-receptor antagonism: studies in man with the enantiomers of chlorpheniramine and dimethindene. Br J Pharmacol. 1991;104(1): 270-276.

21. Harvey AG, Schmidt DA, Scarna A, Semler CN, Goodwin GM. Sleeprelated functioning in euthymic patients with bipolar disorder, patients with insomnia, and subjects without sleep problems. Am J Psychiatry. 2005;162(1):50-57.

22. Palmese LB, DeGeorge PC, Ratliff JC, et al. Insomnia is frequent in schizophrenia and associated with night eating and obesity. Schizophr Res. 2011;133(1-3):238-243.

23. Gao K, Kemp DE, Fein E, et al. Number needed to treat to harm for discontinuation due to adverse events in the treatment of bipolar depression, major depressive disorder, and generalized anxiety disorder with atypical antipsychotics. J Clin Psychiatry. 2011;72(8):1063-1071.
Neuropsychiatric Disease and Treatment

\section{Publish your work in this journal}

Neuropsychiatric Disease and Treatment is an international, peerreviewed journal of clinical therapeutics and pharmacology focusing on concise rapid reporting of clinical or pre-clinical studies on a range of neuropsychiatric and neurological disorders. This journa is indexed on PubMed Central, the 'PsycINFO' database and CAS.

\section{Dovepress}

The manuscript management system is completely online and includes a very quick and fair peer-review system, which is all easy to use. Visit http://www.dovepress.com/testimonials.php to read real quotes from published authors. 